\title{
Investigating Improved Oil Recovery in Heavy Oil Reservoirs
}

\author{
Yogesh Kumar Suman', Ezeddin Shirif ${ }^{1}$, Hussameldin Ibrahim¹, Abdulsalam Ala-Ktiwi² \\ ${ }^{1}$ Faculty of Engineering, University of Regina, Regina, Canada \\ ${ }^{2}$ Faculty of Engineering, University of Tripoli, Tripoli, Libya \\ Email:
}

Received 5 October 2013; revised 7 November 2013; accepted 15 November 2013

Copyright (C) 2014 Yogesh Kumar Suman et al. This is an open access article distributed under the Creative Commons Attribution License, which permits unrestricted use, distribution, and reproduction in any medium, provided the original work is properly cited. In accordance of the Creative Commons Attribution License all Copyrights (C) 2014 are reserved for SCIRP and the owner of the intellectual property Yogesh Kumar Suman et al. All Copyright @ 2014 are guarded by law and by SCIRP as a guardian.

\section{Abstract}

Primary production mechanisms do not recover an appreciable fraction of the hydrocarbon initially in place (HIIP). Practical knowledge has shown that, at the point when the natural energy in a heavy oil reservoir is nearly or altogether depleted, the recovery factor does not exceed about $\mathbf{2 0 \%}$. Some heavy oil reservoirs do not produce at all by natural drive mechanisms. This often necessitates adopting a production improvement strategy to augment recovery. Prior to implementing an improved oil recovery method (either secondary or tertiary) in the field, it is very important to investigate its potential for success. Reservoir simulation is a part of a continuous learning process used to gain insight into the feasibility and applicability of improved oil recovery methods. In this project, GEM compositional reservoir simulator has been used to study the efficiencies of different improved oil recovery strategies, ranging from waterflooding to solvent injection. The drainage volume investigated is a hypothetical box-shaped heavy oil reservoir composed of three distinct permeable layers.

\section{Keywords}

Heavy Oil; IOR; GEM Simulator; Reservoir Simulation; Drainage Volume

\section{Introduction}

Improved oil recovery processes broadly encompass all of the measures aimed towards increasing ultimate recovery from a petroleum reservoir. Most reservoirs are subjected to improved oil recovery (IOR) processes fol${ }^{*}$ Corresponding author. 
lowing primary recovery. Natural reservoir energies control the ultimate recovery of petroleum during primary production; such drive mechanisms include liquid and rock compressibility drive, solution gas drive, gas-cap drive, natural water influx, and combination drive processes. Primary recovery from oil reservoirs is influenced by reservoir rock properties, fluid properties, and geologic heterogeneities.

Methods of improved oil recovery processes are classifiable into two groups: secondary production methods and enhanced oil recovery (EOR) methods. Secondary production methods are based on fluid injection, and they are targeted at providing further energy in order to augment or sustain the production level once well rates decline during primary recovery. Such processes include both water flooding and natural gas injection. Since a considerable amount of oil is left after primary and secondary production methods, the ideal goal of enhanced oil recovery processes is to mobilize the "residual" oil throughout the entire reservoir. This can be achieved by enhancing microscopic oil displacement and volumetric sweep efficiencies. Oil displacement efficiency can be increased by decreasing oil viscosity using thermal floods or by reducing capillary forces or interfacial tension with chemical floods. Processes here consist of all methods that use external sources of energy and/or materials to recover oil that cannot be produced economically by conventional means; they are broadly classified as thermal (steam flooding, hot water flooding, and in situ combustion) and non-thermal (chemical flood, miscible flood, and gas drive). Alternatively, enhanced oil recovery methods are called tertiary oil recovery processes.

The objective of this paper is to compare the productivities of different improved oil recovery methods in a hypothetical box-shaped heavy oil reservoir. Recovery processes considered include water injection, gas injection, and water-alternating-gas (WAG) injection. In each scenario, production by a vertical well is considered separately from that by a horizontal well. The GEM (a component of the CMG suite of reservoir simulators) is used in this study to achieve this comparison. GEM is a general equation-of-state (EOS) based compositional simulator for modelling the flow of three-phase, multi-component fluids. It is effective for modeling any type of reservoir where the importance of the fluid composition and their interactions are essential to the understanding of the recovery process.

\section{Model Description}

The numerical model [1] used in this study is basically a "box" reservoir with a $7 \times 7 \times 3$ rectangular grid pattern corresponding to a volume of $3500 \times 3500 \times 100 \mathrm{cu} \mathrm{ft}$. The reservoir is buried $8325 \mathrm{ft}$ below the ground surface. The reservoir is initially under saturated, and has a constant bubble-point pressure. The permeability characterization is directional both in the reservoir and the aquifer; permeability in the horizontal (x- and $y$-directions) is constant but different from that in the vertical (z-direction). Porosity is also kept constant. The boundaries of the hypothetical reservoir are all no-flow boundaries, and the pressure in the volume is initially uniform. Figure 1 shows the reservoir layers together with the rock and dynamic properties governing fluid flow in the system. The data used in this study are presented in Table 1.

\section{Simulation Scenarios}

GEM (CMG, [2]) was used to model (CMG, [3]) different improved-oil-recovery situations-water, gas and water-alternating-gas injection mechanisms. Each simulation run is done for a production time span of 20 years, and the cardinal production variables (oil production rate, cumulative oil production, water cut, cumulative gas-oil ratio, flowing bottomhole pressure, and average reservoir pressure) are examined. It is assumed that there is no permeability alteration in the vicinity of the producing wells; hence no skin factor is set to zero in each production scenario.

Each simulation run is dedicated to a specific production technique that combines a production well and an injection well. The objective of each run is to evaluate how the injection sustains and improves recovery at the production well. Both the production and injection well are controlled by a set of constraints aimed to keep production going for as long as possible and hence raise the levels of cumulative oil production. Table 2 indicates the constraints set on the production and injection wells.

\section{Case 1: Vertical Production Well}

This is the base case with which the rest cases are compared. It consists of just a vertical production well located at the grid $(7,7,1)$.

Case 2: Vertical Production Well and Vertical Water Injection Well

A vertical production well is located at grid $(7,7,1)$ in the reservoir volume. Fluid withdrawal from the pro- 


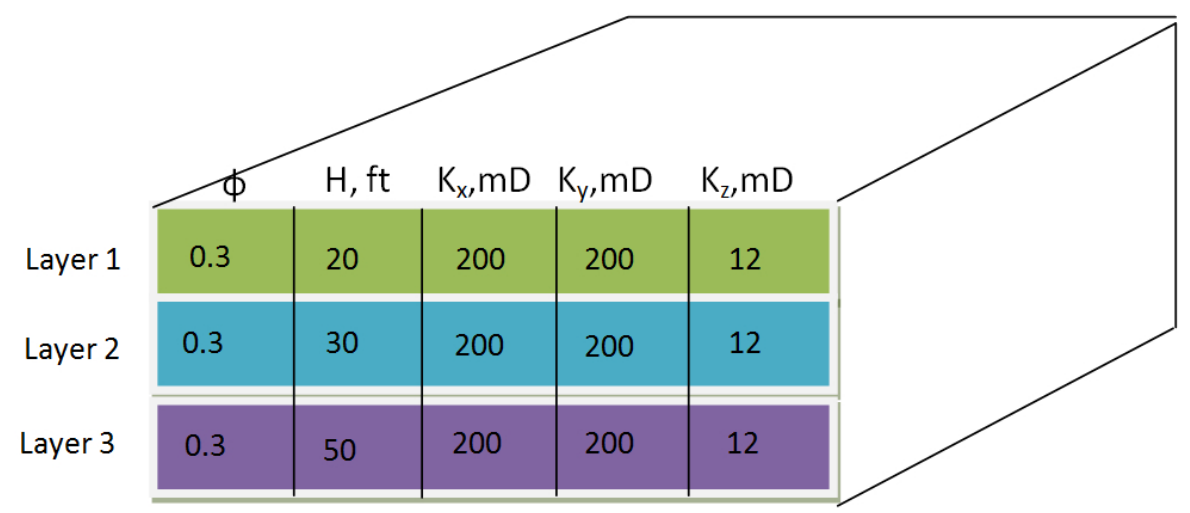

Figure 1. Reservoir system.

Table 1. Table type styles.

\begin{tabular}{cc} 
Reservoir Rock and Fluid Properties Used in Simulation \\
\hline Reservoir depth, ft & 8325 \\
Reservoir thickness, ft & 100 \\
Area of reservoir, sq ft & $1.00 \mathrm{E}+04$ \\
Reservoir rock compressiblity, /psi & $3.00 \mathrm{E}-06$ \\
X-direction permeability, mD & 200 \\
Y-direction permeability, mD & 200 \\
Z-direction permeability, mD & 12 \\
Initial reservoir porosity & 0.3 \\
Initial reservoir pressure, psia & 4800 \\
Oil bubble-point pressure, psia & 500 \\
Reservoir temperature, F & 158 \\
Length of horizontal well, ft & 4243 \\
Well radius, ft & 0.25 \\
Skin & 0 \\
Total simulation time, years & 20 \\
\hline
\end{tabular}

Table 2. Injection and production constraints.

\begin{tabular}{cccc}
\hline Well & Constraint & Value & Action \\
\hline Injector & Bottom hole pressure (maximum) & $4200 \mathrm{psi}$ & Production \\
& Surface oil rate (maximum) & $12,000 \mathrm{bbl} / \mathrm{day}$ & Production \\
Producer & Bottom hole pressure (minimum) & $1000 \mathrm{psi}$ & Production \\
\hline
\end{tabular}

duction well is enhanced by water injection from another vertical well located at grid $(1,1,3)$.

Case 3: Vertical Production Well and Vertical Gas Injection Well

The configuration and location of the production well in this scenario are exactly identical to those in Case 2; the only exception is the injection fluid used. The recovery process here is supported by the injection of gas through a vertical well located at $(1,1,3)$.

Case 4: Vertical Production Well and Vertical WAG Injection Well

In this case, oil recovery is carried using a WAG-injection support mechanism. Both the production and injection wells are vertical and located at grids $(7,7,1)$ and $(1,1,3)$ respectively.

Case 5: Horizontal Production Well

A horizontal production well placed at grid $(4,7,1)$ through $(7,4,1)$ to drain the reservoir volume. The production from the systems is totally aided by the natural energy of the reservoir.

Case 6: Horizontal Production Well and Vertical Water Injection Well 
The architecture and location of the horizontal production well is identical to that in case 5 , but the production is supported by water injection from a vertical well at grid $(1,1,3)$.

Case 7: Horizontal Production Well and Vertical Gas Injection Well

This setup is the same as in case 6; the only difference lies in the injection fluid adopted here. Gas is injected to maintain the pressure in the reservoir and thus enhance recovery.

\section{Case 8: Horizontal Production Well and Vertical WAG Injection Well}

Like case 4 above, water-alternating-gas injection strategy is employed here. The horizontal production well is placed at grid $(4,7,1)$ through $(7,4,1)$ while the vertical injection well is drilled at $(1,1,3)$.

Please see well configuration and architecture in Figure 2.

\section{Discussion of Results}

Figure 3(a) through Figure 3(e) applies to all the production methods relating to the utilization of a vertical production well. Figure 3(a) describes the average reservoir pressure in each system. As can be seen, the water-injection and WAG-injection methods provide the earliest pressure support compared to the other methods; overall, the WAG-injection holds a very high promise of boosting the reservoir pressure appreciably. This pressure phenomenon is translated directly to both the production rate [Figure 3(b)] and cumulative production [Figure 3(c)] from each setup. Within the time span considered in this study, the WAG assisted production mechanism records the highest production rates and cumulative production, followed by the water-injection recovery technique. And as expected, the gas injection method recovers a little above the production level for the base case (vertical production well only), but does not very prove very efficient. The GOR from all the production methods [Figure 3(d)] are considerable except for the base case where the gas-oil ratio takes a steep rise after the 13th year of production. Equally, all the production strategies perform excellently well in terms of watercut [Figure 3(e)] until the 13th year of production after which the production well in the WAG technique washes out with an instant $50 \%$ water production. To boost productivity, the 13th year may likely be the best time to commence a remediation job for the WAG recovery method.

In the horizontal production well scenario, Figure 4(a) through Figure 4(b) explains the production trend. Apart from the WAG method that records appreciable reservoir pressure enhancement, the rest methods do not seem to be potentially efficient to boost pressure [Figure 4(a)]. Therefore, both the gas-injection and water-injection mechanisms exhibit similar pressure trend as the horizontal well base case except for minor deviations noticeable from 1998 and 1999 respectively. This poor performance could be attributed to the location of the horizontal well in the drainage volume; it is too close to two contiguous boundaries of the reservoir (see Figure 2). The observation is not different in the production rates of each method [Figure 4(b)]. In terms of cumulative production [Figure 4(c)], the water injection method shoots ahead the base case marginally while WAG method shows a significant level of additional recovery. The only setback in the WAG recovery technique is in the area of water production. Figure 4(d) shows the gas to oil ratio produced under different scenarios over the simulation time whereas Figure 4(e) reveals that water breakthrough occurs at the production well after about 11 years.
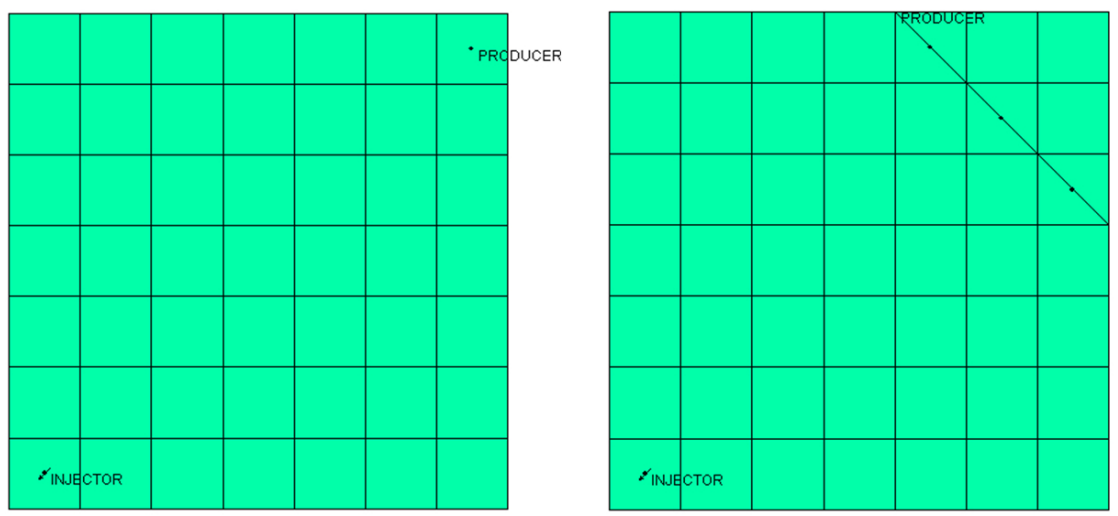

Figure 2. Well architecture and location (aerial view). 


\begin{tabular}{|l|l|}
\hline \multicolumn{2}{|c|}{ Key } \\
\hline VertWell & Vertical well only \\
\hline VertWell_WaterInjt & Vertical well and water injection well \\
\hline VertWell_GasInjt & Vertical well and gas injection well \\
\hline VertWell_WaterInjt & Vertical well and WAG injection well \\
\hline HorizWell & Horizontal well only \\
\hline HorizWell_WaterInjt & Horizontal well and water injection well \\
\hline HorizWell_GasInjt & Horizontal well and gas injection well \\
\hline HorizWell_WaterInjt & Horizontal well and WAG injection well \\
\hline
\end{tabular}

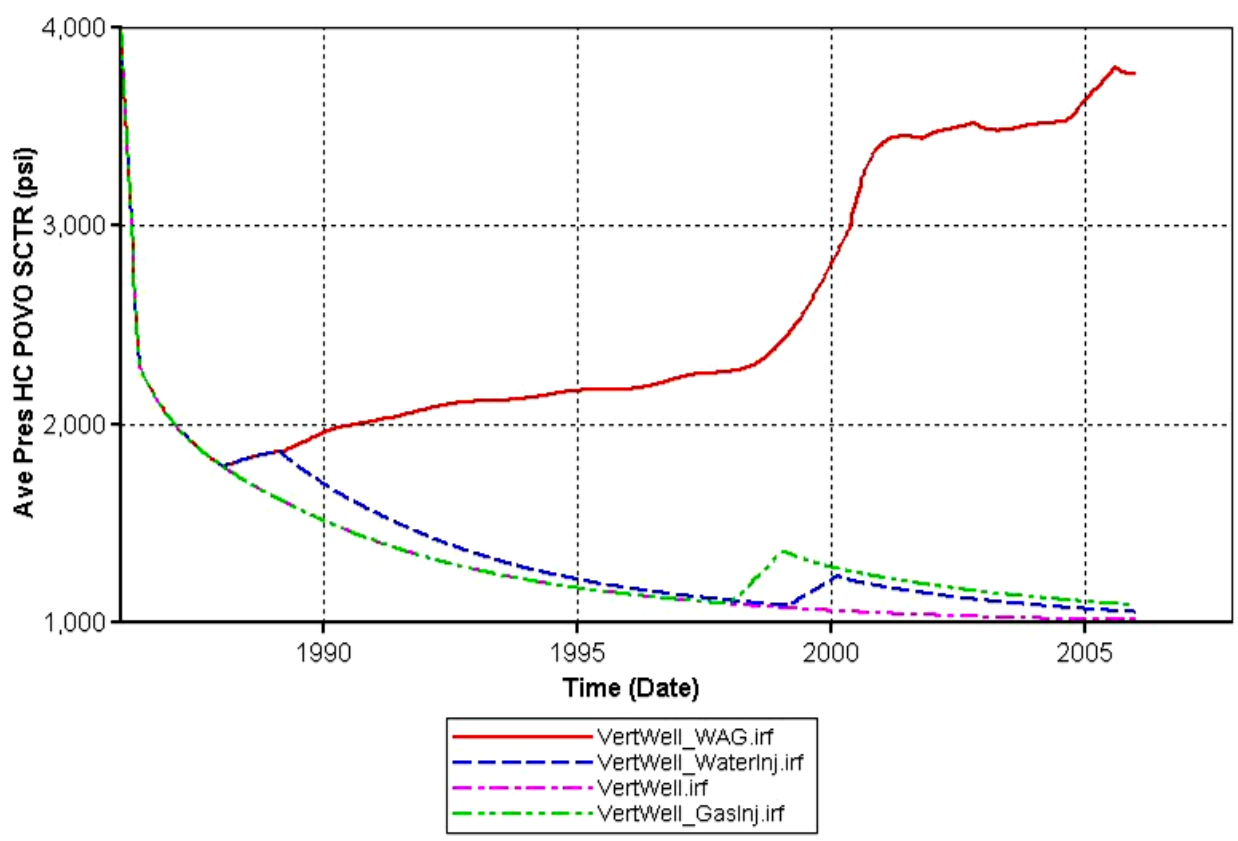

(a)

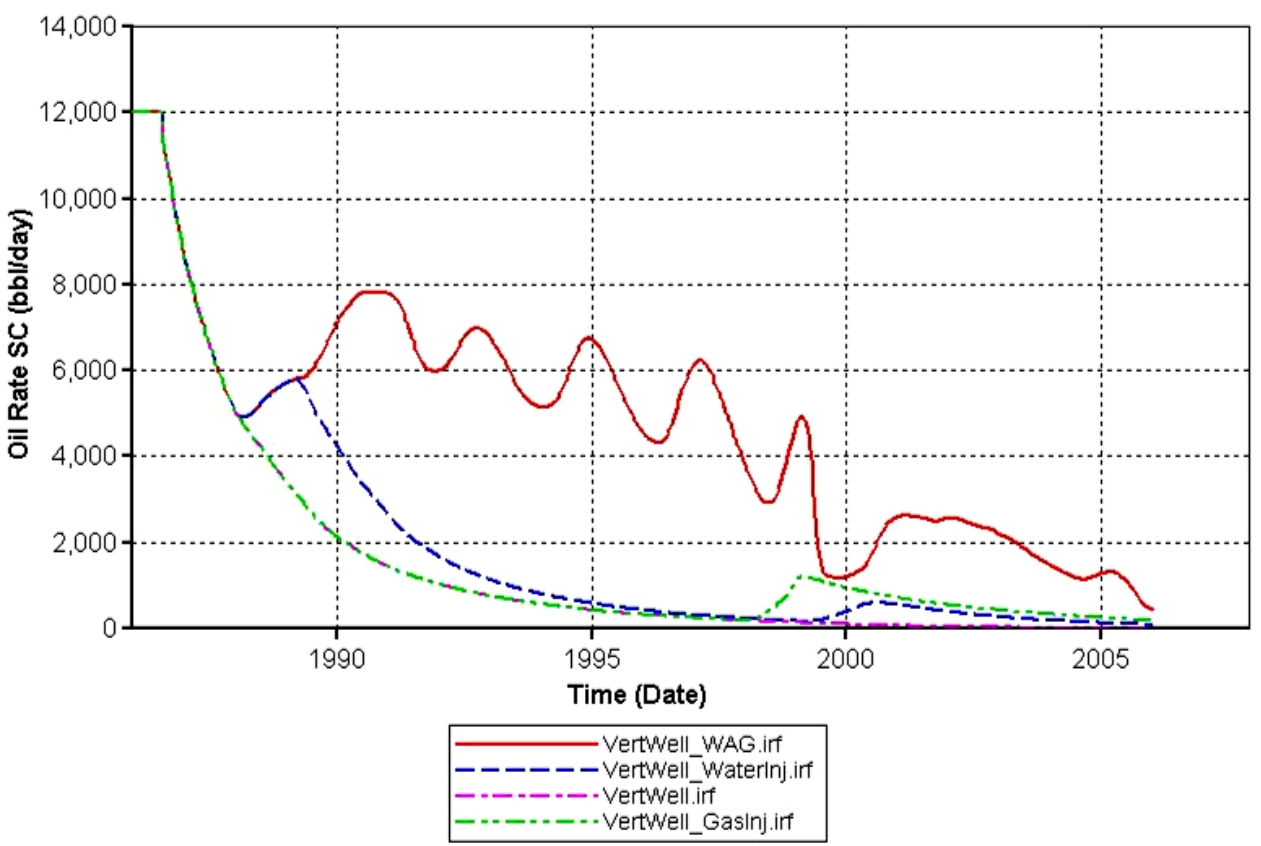

(b) 


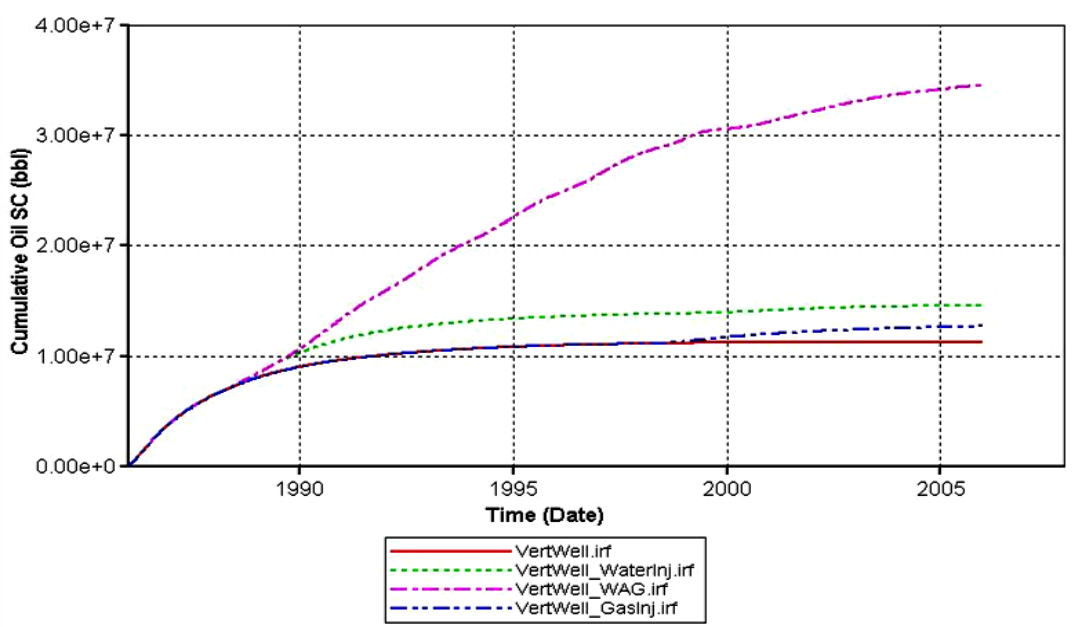

(c)

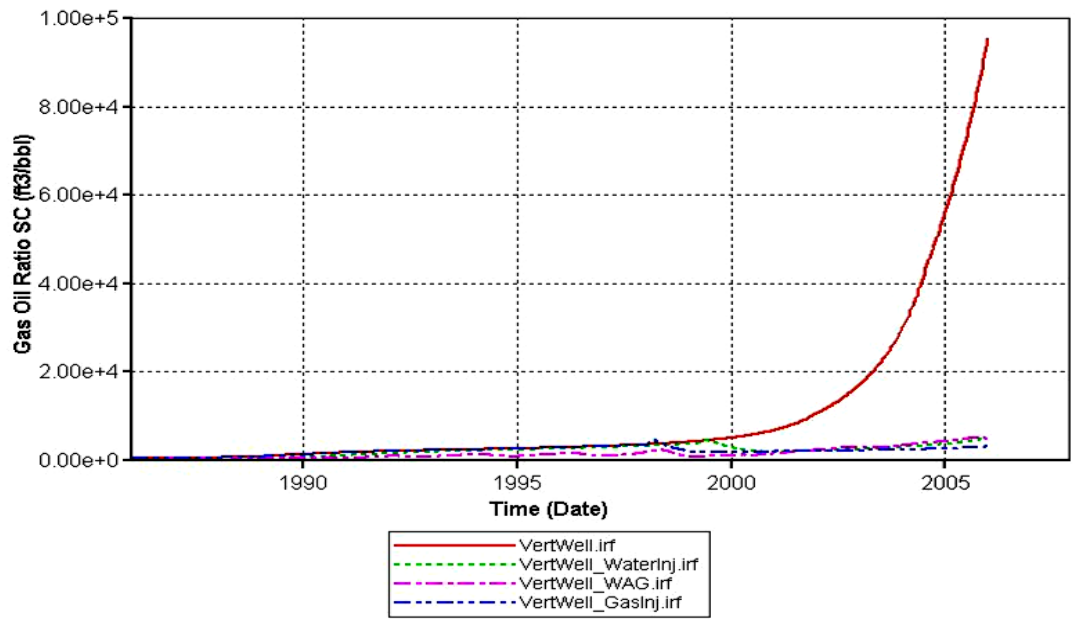

(d)

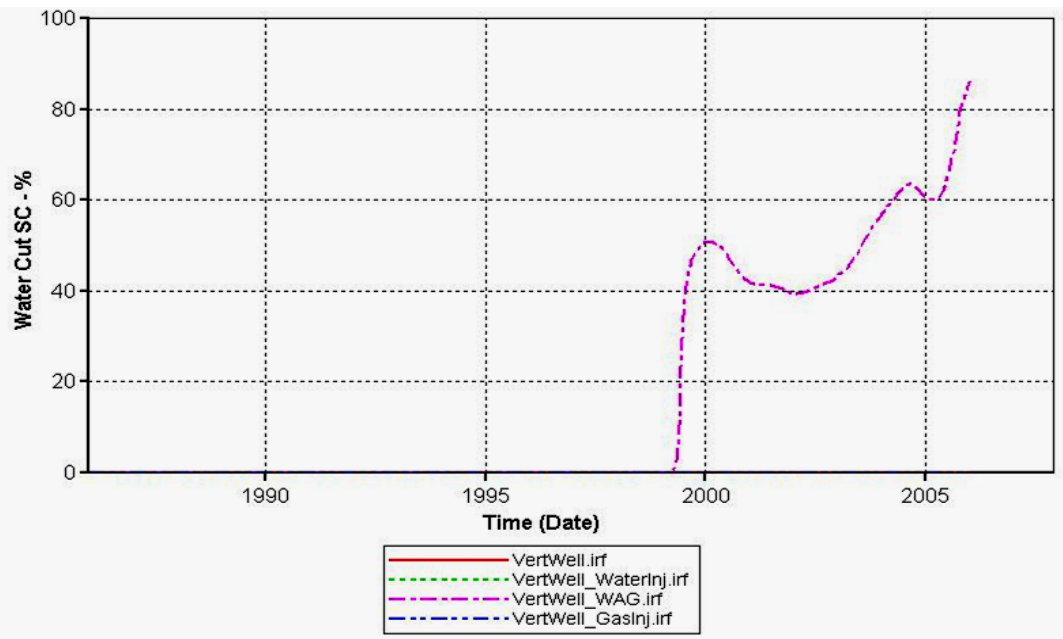

(e)

Figure 3. For plots shown in Figure 3(a) through Figure 4(e). (a) Average reservoir pressure; (b) Surface oil production rate; (c) Cumulative stock tank oil; (d) Gas-oil ratio; (e) Water cut. 


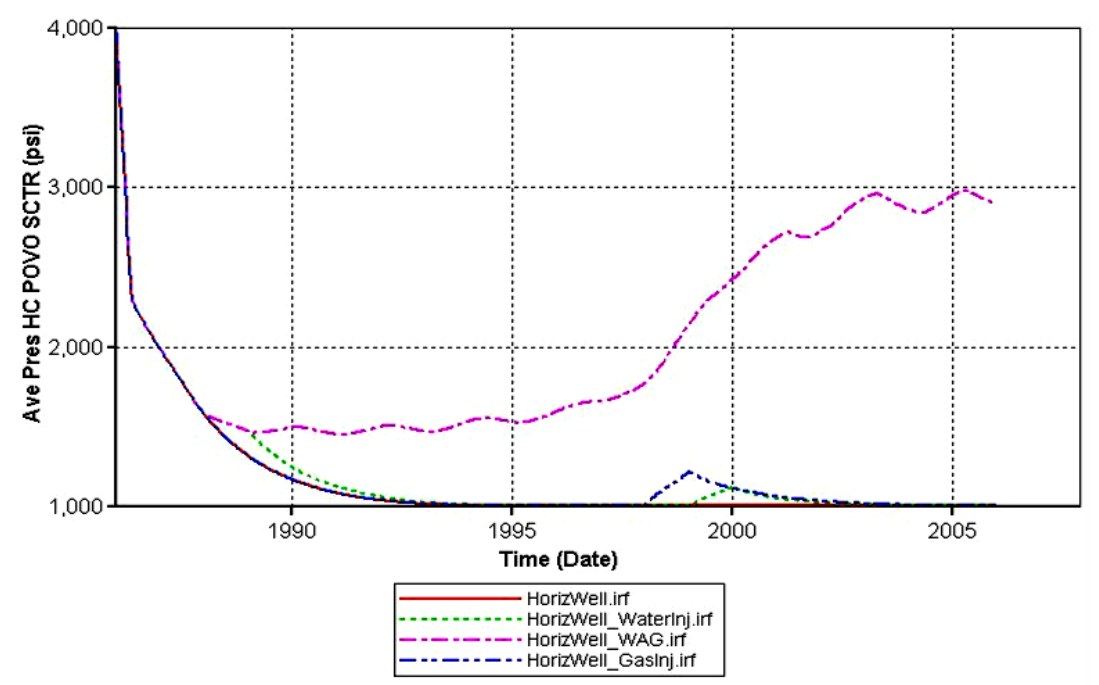

(a)

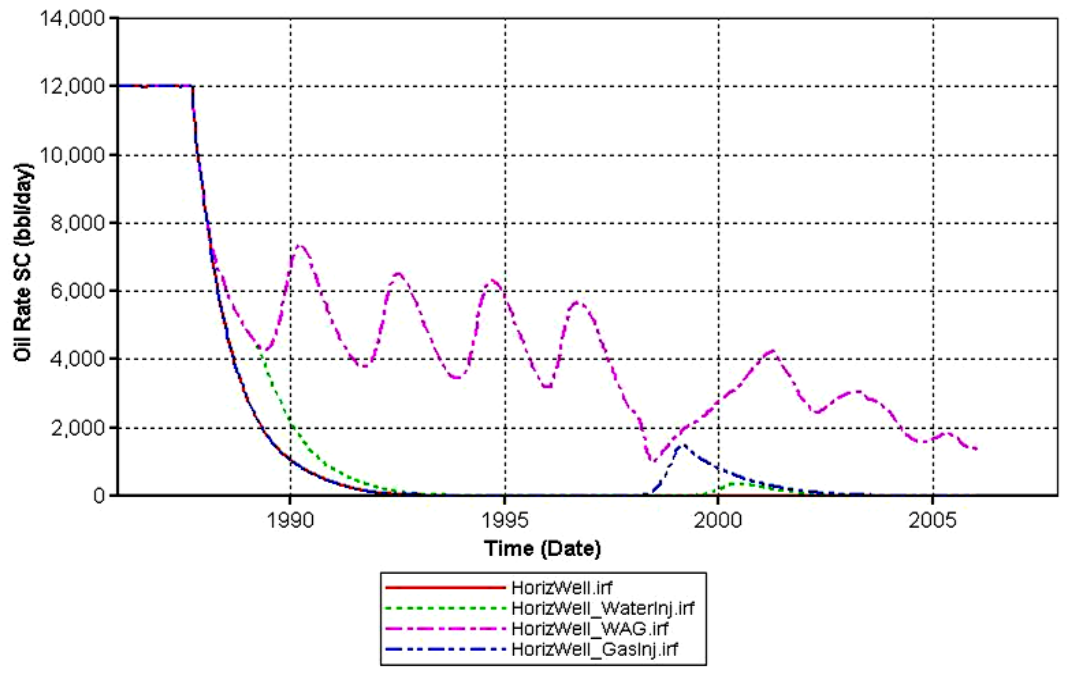

(b)

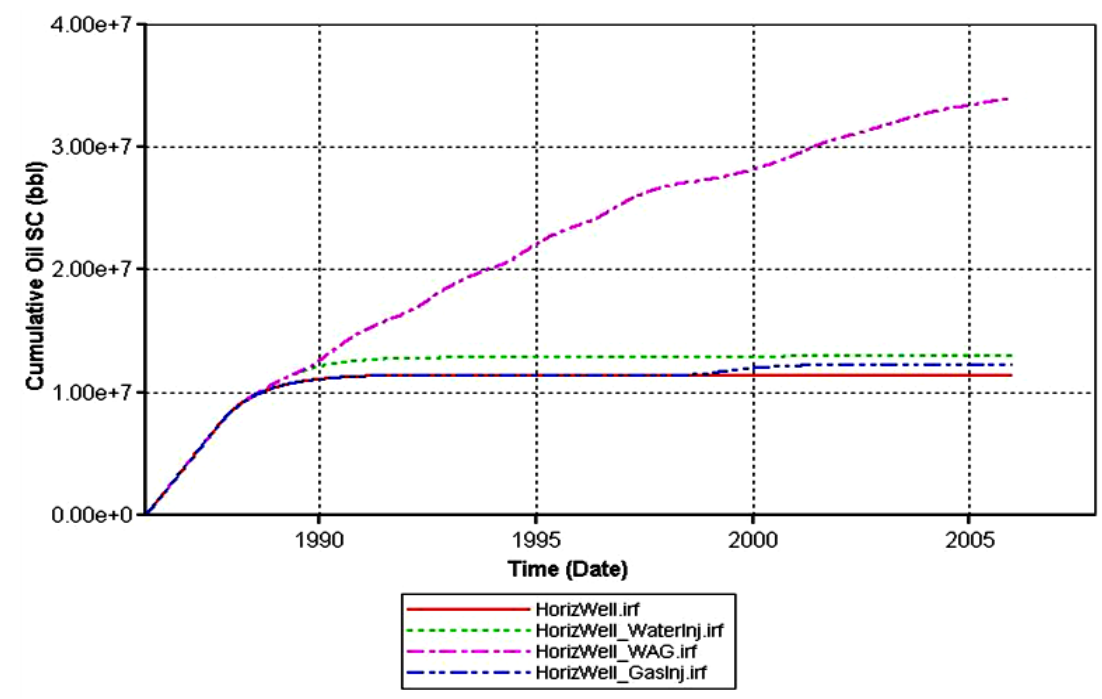

(c) 


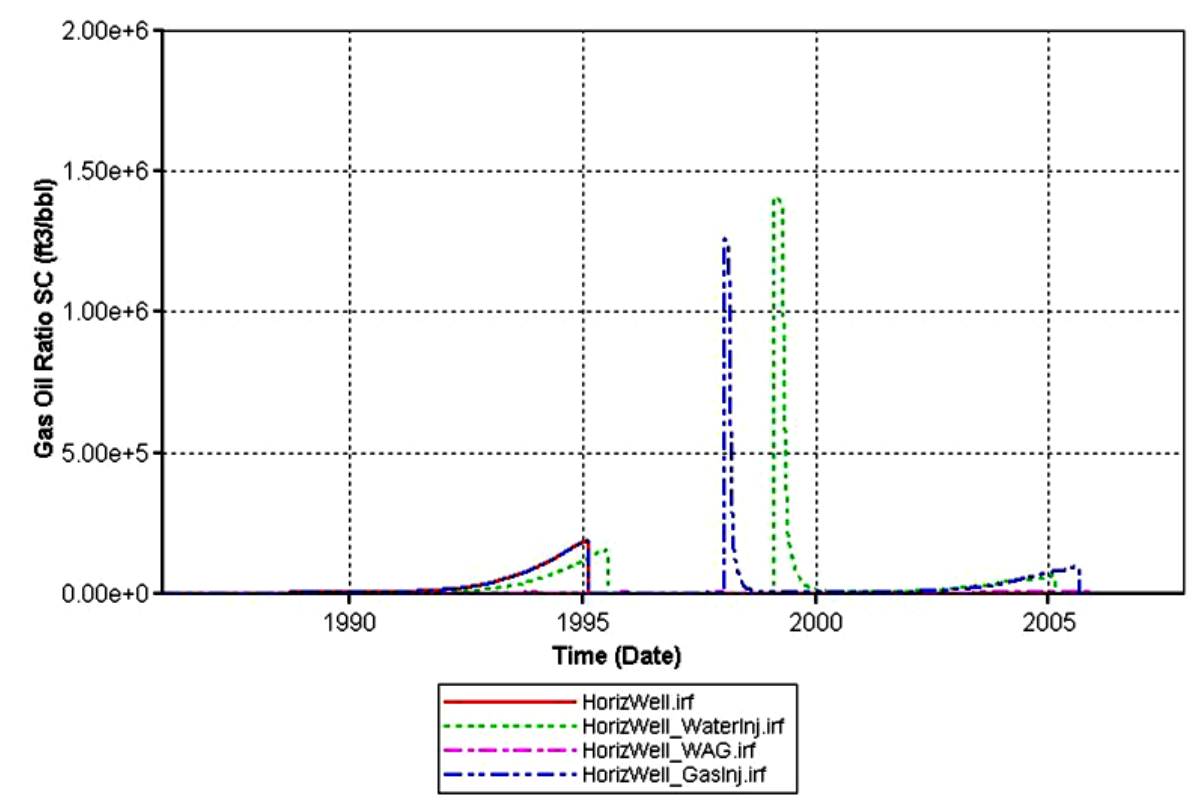

(d)

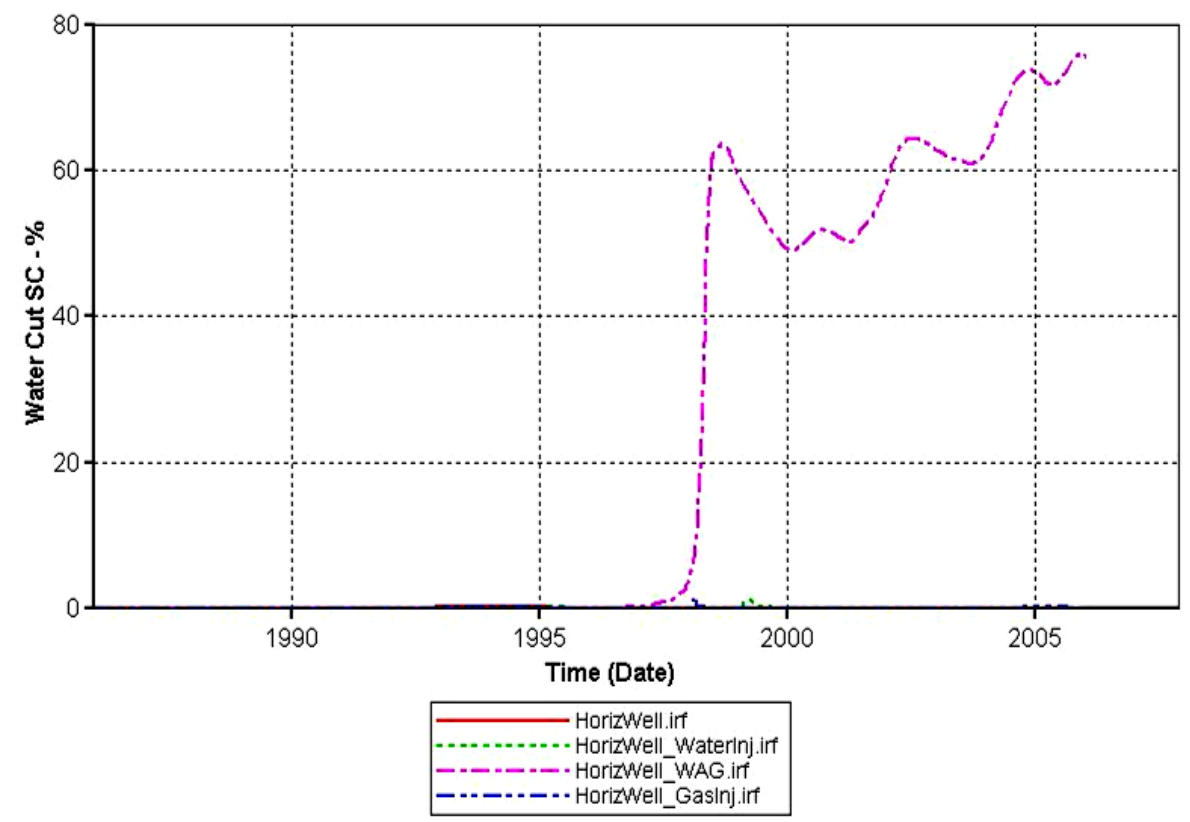

(e)

Figure 4. (a) Average reservoir pressure; (b) Surface oil production rate; (c) Cumulative stock tank oil; (d) Gas-oil ratio; (e) Water cut.

\section{Conclusions}

This study only investigates the additional recovery accruable to the implementation of improved oil recovery techniques (three methods are studied here). It does not consider the effect of well placement, length of horizontal well or distance between production and injection wells.

Of all three methods studied, the WAG recovery technique shows the greatest potential of optimizing recovery. The water-injection method performs relatively better than the gas-injection method.

It is not logical to draw a conclusion on the precedence of vertical/horizontal production well over the other 
until an optimum well configuration is obtained for each well type. This is beyond the scope of this study.

\section{References}

[1] Aziz, S.O. (1981) Comparison of solutions to a three-dimensional black-oil reservoir simulation problem. Paper SPE 9723. http://www.ipt.ntnu.no/ kleppe/pub/SPE-COMPARATIVE/papers/first.pdf

[2] Computer Modelling Group (2003) Compositional simulation using GEM.

[3] Computer Modelling Group (2007) Building, running and analyzing a black oil reservoir simulation model using Builder 2007.1 and IMEX 2007.1. 